\title{
Transformation du mode de pensée et d'action des médecins à l'hôpital
}

\section{Richard Otto Binswanger}

Médecin-chef radiologue de l'établissement Spital Thurgau AG Münsterlingen et président de la Thurgauische Vereinigung Leitender Spitalärzte (Société thurgovienne des médecins-chefs en hôpital)
Correspondance:

Dr. med. R. O. Binswanger

STG AG

Kantonsspital

Radiologie

CH-8596 Münsterlingen

Tel. 0716862330

Fax 0716862647

richard.binswanger@stgag.ch

www.vlss.ch

\section{Introduction}

Jusqu'à récemment, dans les hôpitaux publics de Suisse, le mode de facturation appliqué aux patients bénéficiant de l'assurance générale suivait presque toujours le principe du forfait journalier. Ce système est en train de changer. A l'avenir, l'indemnisation de la prestation hospitalière se fera suivant les Diagnosis-related Codes (DRG). Elle sera perçue sous forme de forfait pour la totalité du séjour hospitalier et son montant variera en fonction du diagnostic du patient. Ainsi, le forfait facturé à un patient victime d'un infarctus ne sera pas le même que celui d'un patient souffrant d'une appendicite aiguë. Les calculs se feront en fonction de la durée moyenne d'hospitalisation et de la mobilisation du personnel et des appareils de l'hôpital. La motivation politique de cette transformation réside dans les coûts élevés, dont on souhaite limiter la progression.

Le système des DRG est apparu dans les pays anglo-saxons et vient d'être introduit en Allemagne. En comparaison, la Suisse est en retard mais cela lui permet d'intégrer les expériences étrangères sur le processus décisionnel politique. Les chefs de service auront désormais un rôle de management plus important, notamment ils assumeront la responsabilité de leur budget et d'une bonne gestion.

Werner Vogd, privat-docent dans le domaine de la «recherche qualitative en éducation» de l'Université libre de Berlin, a publié en 2006 une monographie portant le titre suivant: «Die Organisation Krankenhaus im Wandel» (l'hôpital comme organisation en transformation) [1]. Son ouvrage est basé sur des enquêtes menées dans les grands hôpitaux de Berlin, au fil d'un accompagnement de médecins de différents secteurs, pendant plusieurs semaines. Leur gestion des patients, des relations avec leurs collègues et avec le reste du personnel a été consignée. Des interviews ont également été menées. Ce travail couvre une période d'observation partant de l'année 2000, ensuite comparée à l'année 2004.
Les DRG furent introduits en 2003. Il s'agit donc d'une observation très précise du mode de pensée et d'action du milieu médical dans les nouvelles conditions.

\section{Résultats}

Avec les DRG, la durée d'hospitalisation du patient occupe une place centrale dans le respect du budget fixé. On vise en même temps une augmentation du nombre de patients traités, afin de compenser les éventuelles pertes de recettes. Le recours à des appareils d'examens onéreux est très critiqué car il grève le budget de l'hôpital qui les prescrit. Les plus lucratifs sont les patients présentant des tableaux cliniques simples, appelant des interventions faciles et sans complication, qui permettent de les libérer rapidement. Tous les autres patients sont considérés par le personnel médical comme non rentables, ce qu'on leur fait sentir de façon plus ou moins directe.

Les services sont soumis à une forte pression de rationalisation à laquelle ils répondent par une décomposition du processus de diagnostic et de traitement. Dans la mesure du possible, les examens avant les interventions sont avancés pour être faits en ambulatoire, tout comme l'examen d'entrée. Les chirurgiens font constamment la navette entre la salle d'opération, les services des patients et les services d'admission et sont à tout moment rappelés de leur lieu de travail, si bien que la tâche entamée ne peut être terminée et doit être déléguée aux collègues.

Les hôpitaux fournissent aux médecins des moyens informatiques pour les aider. Aujourd'hui, chacun a son ordinateur. La quantité de tâches administratives devant être gérées sur ordinateur a considérablement augmenté. Pour la facturation, à chaque patient doit correspondre un ou plusieurs codes diagnostic. Les délais des tâches administratives sont fixés dans les systèmes informatiques, de même que ceux des rapports, etc. et sont ensuite surveillés par le système. 
Ainsi, dès qu'ils ont une minute de libre, les médecins se mettent à l'ordinateur pour régler les tâches administratives dans les délais. Le travail qui n'est pas contrôlé de façon formelle souffre de ce contexte. Notamment le temps disponible pour le patient est considérablement réduit. Ce phénomène est perçu comme une entrave à la relation médecin-patient. Afin d'optimiser les procédures de travail dans les cas routiniers, on fait de plus en plus appel au diagnostic standard. Les décisions sont prises au vu du dossier et non plus en «face à face» avec le patient.

La limitation du temps de travail, réduit à moins de cinquante heures pour les médecinsassistants et les chefs de clinique, entre aussi en ligne de compte. Elle renforce l'absence de continuité dans les soins apportés au patient. En cherchant à raccourcir la durée d'hospitalisation, en cas de diagnostic douteux, on a de plus en plus recours aux appareils, même s'il n'est pas certain que l'examen soutienne le diagnostic.

Les médecins-assistants notamment ne peuvent plus remplir complètement leur mission, ce qui a entraîné un changement informel des priorités dans les hôpitaux. Ainsi, on accepte des pertes de qualité dans le suivi du dossier médical ainsi que de la suppression de la visite de médecin systématique le week-end et les jours de fête. Les patients dont le tableau clinique ne présente pas un caractère d'urgence sont placés en division hospitalière sans examen plus approfondi et ne sont auscultés de près par le médecin que le lundi suivant. On concentre son attention sur les «cas difficiles» avec un diagnostic peu clair, des symptômes de maladie supplémentaires avant les interventions et des complications. Étant donné la fragmentation de l'action médicale, le plus grand nombre de médecins possible doivent connaître ces cas afin de pouvoir réagir à tout moment.

Dans un contexte de réductions budgétaires, l'interdisciplinarité devient problématique car les réunions avec des spécialistes d'autres divisions entraînent des coûts. Comme on ne peut compter qu'une fois les forfaits DRG au cours du même séjour, lorsque le patient change de service au sein de l'hôpital, cela entraîne des réflexions laborieuses. Les patients sont transférés très tôt vers les institutions subordonnées, même si le traitement n'est pas encore complètement terminé. Les médecins sont pourtant tout à fait conscients que ces institutions, par exemple les EMS, sont moins bien équipées que les hôpitaux pour le traitement du tableau clinique. Il est frappant de constater que d'importants efforts sont déployés pour maintenir la qualité des soins médicaux. Bien que menacés, les principes de l'éthi- que médicale passant par une gestion la plus globale possible du patient sont toujours respectés lorsque c'est encore possible.

La pression en faveur de la rationalisation a entraîné un nivellement de la hiérarchie médicale et les médecins-chefs sont maintenant plus facilement joignables qu'avant, mais au détriment d'une gestion autonome du temps des chefs de service. Ils ne peuvent plus décider euxmêmes des questions qu'ils abordent et doivent être prêts à tout moment à prendre des décisions. Toujours dans le cadre de ce processus d'adaptation, les moyens techniques comme le téléphone mobile et l'ordinateur sont bien plus utilisés qu'avant. On s'achemine globalement vers une augmentation de l'efficacité.

\section{Evaluation des résultats}

Dans les cliniques observées, l'introduction des DRG et la responsabilité budgétaire des chefs de service, associées aux restrictions budgétaires et aux réductions du temps de travail ont imposé une pression énorme pour pouvoir s'adapter. La durée d'hospitalisation des patients est la priorité des priorités et en même temps le nombre de patients à traiter doit augmenter: ce défi ne peut être relevé qu'avec de nouvelles formes d'organisation. Le partage du travail dans le processus de traitement augmente, tout est décomposé. Les examens préliminaires sont transférés en ambulatoire. Le traitement est poursuivi dans des institutions subordonnées, moins bien adaptées. Le contact médecin-patient est réduit au minimum, standardisé et les appareils de diagnostic sont de plus en plus utilisés. L'attention des médecins est concentrée sur les patients difficiles, en acceptant de perdre en qualité dans le suivi des cas simples et dans la gestion du dossier médical, ainsi que de réduire l'interdisciplinarité. Le système ne s'est pas encore stabilisé dans les hôpitaux observés. A ce jour, les pertes de qualité n'ont pas pu être compensées par des mesures organisationnelles.

Les institutions optimisent leur propre budget. La question de savoir si l'incidence est positive sur le coût global de la santé publique n'est pas encore tranchée. Il est notamment remarquable que soit maintenu le respect des exigences de l'éthique médicale, appelant une médecine globale et adaptée au patient. Cependant, cette éthique est perçue comme fortement menacée.

\section{Les conséquences en Suisse}

En Allemagne, le système de santé est soumis à une pression économique bien plus forte qu'en Suisse. On assiste actuellement à un exode des 
médecins et du personnel qualifié non médical qui quittent l'Allemagne, affichant ainsi clairement leur opinion. En Suisse, le monde politique pense aussi résoudre les problèmes grâce aux DRG et à la responsabilisation budgétaire. En outre, les systèmes d'assurance qualité et de réseaux doivent aider à assurer les soins. Quant à la réduction du temps de travail des médecins, c'est déjà une réalité en Suisse.

Les médecins des hôpitaux suisses sont responsables de la mise en ouvre des nouvelles directives économiques. Leur mission est d'apporter aux patients des soins globaux et adaptés. Ils doivent avoir des pouvoirs décisionnels donnant généralement la priorité aux considérations médicales et non économiques. Cette mission ne peut être remplie qu'à condition que la position des médecins-chefs dans la gestion des hôpitaux soit renforcée à la mesure de leur responsabilité et non affaiblie. Cela implique une inversion de la tendance actuelle, qui ne pourra devenir réalité qu'à condition d'être résolument exigée par les médecins des hôpitaux. Il ne reste plus qu'à espérer que la politique et les autres groupes d'interlocuteurs du secteur de la santé reconnaissent cela comme un progrès.

\section{Référence}

1 Vogd W. Die Organisation Krankenhaus im Wandel. Document d'évaluation du point de vue des acteurs du milieu médical. Berne: Huber; 2006.

\section{Extraits de la monographie de W. Vogd}

Dans le quotidien des services hospitaliers, on distingue désormais nettement les cas compliqués et problématiques de ceux qui relèvent de la routine. Contrairement à ce qui se passait avant, ces derniers ne sont plus examinés que rapidement. Les médecins-assistants prennent leur parti de failles dans les soins prodigués et étant donné le temps limité dont ils disposent, les chefs de clinique font très peu attention à ces patients. De plus, le temps réservé à l'entretien entre le médecin et le patient est énormément réduit, de même que les plages horaires réservées à la communication entre médecins. Les visites se font de moins en moins en groupe et le relais se fait généralement par écrit, sans commentaire oral complémentaire.

Maintenir l'équilibre entre les exigences médicales et organisationnelles relève sans aucun doute d'un difficile parcours du funambule, à faire au pas de course. Il faut prendre son parti de certaines lacunes, pallier les discontinuités de personnel, tout en ayant de moins en moins de temps pour transférer les informations et en gérant les risques et erreurs de management inhérents, afin de réparer les failles qui en

Conséquences des DRG et de la responsabilisation budgétaire en quelques mots-clés

- La réduction de la durée d'hospitalisation devient la plus grande priorité.

- Pour compenser, augmentation du nombre de patients traités.

- Les patients sont considérés comme source de profits ou de pertes.

- Une décomposition systématique du travail conduit à une fragmentation de la chaîne de traitement.

- Examens préliminaires en ambulatoire.

- Priorité à l'exécution des tâches administratives soumises à des délais, par rapport au travail avec le patient.

- Moins de temps médical passé avec le patient.

- Absence de continuité dans la responsabilité envers le patient.

- Diagnostics standard et décisions d'après dossiers dans les cas routiniers.

- Recours de plus en plus fréquent aux appareils de diagnostic.

- Gestion informelle des priorités par le personnel médical subordonné, avec

- pertes de qualité dans le suivi du dossier médical,

- suppression de la visite systématique du médecin le week-end et les jours fériés.

- Concentration de l'attention sur les «cas difficiles».

- Perte de l'interdisciplinarité.

- Réflexions laborieuses en cas de transfert des patients au sein du même hôpital.

- Transfert des patients dans des institutions subordonnées avant la fin du traitement.

- Nivellement de la hiérarchie médicale et perte d'autonomie dans la gestion de leur temps pour les chefs de service.

- Développement de I'utilisation des moyens techniques comme les téléphones mobiles ou les PC.

- Malgré tout, mobilisation importante en faveur de décisions globales, adaptées aux patients.

- Attribution des erreurs aux médecins en particulier et non au système. 
découlent inévitablement. Etant donné la priorité donnée aux durées d'hospitalisation courtes, ces procédures se déroulent dans un laps de temps bien plus limité. Comme l'orientation médicale n'a pas le droit de se tromper, il n'en résulte pas moins de médecine, mais une médecine plus rapide.

On pourrait constater que pour respecter la primauté des durées d'hospitalisation courtes, notamment dans les cas complexes, les médecins utilisent en parallèle des procédés de diagnostic plus chers et plus rapides. Il est possible qu'on exerce alors une médecine onéreuse, souvent évaluée après-coup comme inutile, dont les frais pourraient être plus élevés que ceux des soins qui seraient facturés pour une durée d'hospitalisation plus longue.

Notamment pour les internistes, l'économie d'intervention médicale a conduit à un «triage» ayant l'effet inverse. Comme les complications et les urgences ont la priorité, les patients présentant des problèmes plus légers sont laissés sans soins pendant une journée, voire un week-end. La limitation des ressources en «forces vives médicales» entraîne un allongement médicalement inutile de la durée d'hospitalisation.

Quelques indices empiriques semblent indiquer que cette réduction drastique de la durée d'hospitalisation ne se fait qu'au prix d'une médecine à base d'équipements plus onéreux, qui doit être exercée par des spécialistes hautement qualifiés. Si l'on observe les expériences faites dans ce domaine aux Etats-Unis, on constate que la réduction de la durée moyenne d'hospitalisation des patients ne conduit pas forcément à une réduction des frais de traitements dans les services.

[...] il ne faut plus exclure le danger que les services médicaux ne fonctionnent plus si les spécialistes désertent les services fonctionnels. On pourrait bien arriver à un tournant où les médecins passent du rôle de personnes tolérantes à la position de revendicateurs.

De plus, de lourdes failles s'annoncent au niveau de la Corporate identity des hôpitaux. Si l'on ne prend pas ce concept comme un affichage formel de modèles, comme la représentation graphique d'emblèmes, qu'on ne le réduit pas à des brochures clinquantes et à des sites Web mais qu'on l'entend bien comme l'identification des collaborateurs à la culture de leur entreprise, alors la situation doit être considérée comme vraiment dramatique. Les élites du corps médical, qui incarnent l'identité médicale et scientifique, n'ont quasiment plus l'impression de faire partie de l'organisation pour laquelle elles travaillent. 